\title{
Cutaneous impact location: a new tool to predict intracranial lesion among the elderly with mild traumatic brain injury?
}

\author{
Xavier Dubucs ${ }^{1 *}$ D, Frederic Balen ${ }^{1}$, Eric Schmidt ${ }^{2}$, Mathieu Houles ${ }^{3}$, Sandrine Charpentier ${ }^{1,3}$, \\ Charles-Henri Houze-Cerfon ${ }^{1}$ and Dominique Lauque ${ }^{1}$
}

\begin{abstract}
Background: Mild traumatic brain injury is the leading cause of arrivals to emergency department due to trauma in the 65-year-old population and over. Recent studies conducted in ED suggested a low intracranial lesion prevalence. The objectives of this study were to assess the prevalence and risk factors of intracranial lesion in older patients admitted to emergency department for mild traumatic brain injury by reporting in the emergency department the precise anamnesis of injury and clinical findings.

Methods: Patients of 65 years old and over admitted in emergency department were prospectively included in this monocentric study. The primary outcome was the prevalence of intracranial lesion threw neuroimaging.

Results: Between January and June 2019, 365 patients were included and 66.8\% were women. Mean age was 86.5 years old $(S D=8.5)$. Ground-level fall was the most common cause of mild traumatic brain injury and occurred in 335 patients (91.8\%). Overall, 26 out of 365 (7.2\%) patients had an intracranial lesion. Compared with cutaneous frontal impact (medium risk group), the relative risk of intracranial lesion was 2.54 (95\% Cl 1.20 to 5.42) for patients with temporoparietal or occipital impact (high risk group) and 0.12 (95\% Cl 0.01 to 0.93 ) for patients with facial impact or no cutaneous impact (low risk group). There was not statistical increase in risk of intracranial injury with patients receiving antiplatelets ( $\mathrm{RR}=1.43 ; 95 \% \mathrm{Cl} 0.68$ to 2.99 ) or anticoagulants ( $\mathrm{RR}=0.98 ; 95 \% \mathrm{Cl} 0.45$ to 2.14 ).

Conclusion: Among patients of 65 years old and over, the prevalence of intracranial lesion after a mild traumatic brain injury was similar to the younger adult population. The cutaneous impact location on clinical examination at the emergency department may identify older patients with low, medium and high risk for intracranial lesion.
\end{abstract}

Keywords: Elderly, Mild traumatic brain injury, Epidemiology, Emergency department

\section{Introduction}

Mild traumatic brain injury (mTBI) is the leading cause of arrivals to emergency department (ED) due to trauma in the 65-year-old population and over [1,2]. Lately, elderly visits to ED for mTBI have increased disproportionately,

\footnotetext{
*Correspondence: xavier.dubucs@gmail.com

'Emergency Department, Centre Hospitalo-Universitaire de Toulouse, Toulouse, France

Full list of author information is available at the end of the article
}

mTBI in the elderly is associated with an increase in morbidity and mortality. This is a frequent reason for hospitalization and it is associated with an alteration in functional and cognitive capacities [2, 3]. Current international guidelines are consistent with the large indication of non-contrast head computed tomography scan (head CT-scan) after mTBI in patients over 65 years old, even without initial consciousness loss $[4,5]$. Head CT-scan is also recommended in all patients under antiplatelets or anticoagulants after mTBI. Former cohorts from which

(C) The Author(s). 2020 Open Access This article is licensed under a Creative Commons Attribution 4.0 International License, which permits use, sharing, adaptation, distribution and reproduction in any medium or format, as long as you give appropriate credit to the original author(s) and the source, provide a link to the Creative Commons licence, and indicate if changes were made. The images or other third party material in this article are included in the article's Creative Commons licence, unless indicated otherwise in a credit line to the material. If material is not included in the article's Creative Commons licence and your intended use is not permitted by statutory regulation or exceeds the permitted use, you will need to obtain permission directly from the copyright holder. To view a copy of this licence, visit http://creativecommons.org/licenses/by/4.0/. The Creative Commons Public Domain Dedication waiver (http://creativecommons.org/publicdomain/zero/1.0/) applies to the data made available in this article, unless otherwise stated in a credit line to the data. 
most international guidelines were derived showed that the intracranial lesions prevalence after mTBI in the elderly was higher than in younger subjects. Regarding to this population, intracranial lesion increased until 30\% [6]. These guidelines are also discussed with specific elderly epidemiological and physiological features. Older patients showing less specific sign of intracranial lesion following head trauma compared to younger patients, they also present less of signs of intracranial hypertension. As well, the Glasgow score is less sensitive and the event taking place during the mTBI is often missing $[7,8]$.

Recent studies conducted in ED suggested a low intracranial lesion prevalence reaching $2.2 \%$ and a rate of neurosurgery lower than $1 \%$ [9]. These data suggest that the brain CT-scan indication following mTBI in this population could be more targeted. In addition to the cost issue, head CT-scan overuse could also have an impact on the patient in terms of radiation-induced neoplasia and cataracts [10]. A prospective study containing detailed injury history and clinical findings at the ED is required to better characterize prevalence and potential risk factors of intracranial lesions.

Our objectives were to assess the prevalence of mTBIrelated intracranial lesions in subjects over 65 years old admitted to the ED and to identify risk factors for intracranial lesions by recording precise mTBI anamnesis as well as clinical findings.

\section{Method}

\section{Study design}

We conducted a prospective descriptive observational study in the two urban University Hospital EDs. These two EDs treated 78,000 and 40,000 patients in 2018 respectively, they located in the middle of a health care pool of approximately 1 million inhabitants.

Between January 2019 and June 2019, all consecutively patients aged 65 years old and older with mTBI admitted to the ED were included before head CT-scan. mTBI was defined as a traumatic brain injury with Glasgow score of 13 or higher on arrival in the ED. Decision to perform head CT-scan was made by the treating physician according to current national guidelines [11]. Patients without head CT-scan performed within the first $24 \mathrm{~h}$ after ED entry were excluded.

Based on the standardized questionnaire filled out in the ED, different types of residence place were reported: living alone at home, with relatives at home (defined by the presence of at least one relative living at home, e.g. wife; child/children) or in a nursing home $(\mathrm{NH})$ ). Antiplatelet (aspirin, clopidogrel), anticoagulants (warfarin, direct oral anticoagulant (Xa/IIa inhibitors) or subcutaneous anticoagulant) and psychoactive drugs were also recorded (benzodiazepine, antidepressant, neuroleptic, antiepileptic). History of neurosurgical intervention and cognitive impairment were included. The patient's frailty was assessed by the Clinical Frailty Scale from 1 (very fit) to 7 (fully functionally dependent) [12]. mTBI was described by the time of the event, witness presence and the injury kinetic (ground-level fall, $>1 \mathrm{~m}$ or 5 steps, road accident, head striking by an object). In case of fall event, the precipitating factor was described: mechanical, faintness or vertigo, syncope. The transient symptoms after the injury were also reported (loss of consciousness, vomiting, seizure, amnesia, headache) and activity after falling (getting up alone or with assistance, staying on floor $>1 \mathrm{~h}$ ). Unavailable variables after patient, witness, or NH requests were categorized as "unknown". Glasgow score, focal neurological signs, basal skull fracture signs (otorrhagia, otorrhea, bilateral periorbital ecchymosis) and cutaneous injury types (cutaenous abrasion, hematoma, wound requiring suture, no cutaneous lesion) were recorded by the treating physician. He also showed the cutaneous impact location on a head figure. The cutaneous impact location was then categorized by the investigator as follow: frontal, temporoparietal, occipital, facial, or no cutaneous impact. After the ED visit, the patient's outcome was noticed: discharge or hospitalization. According to ED and hospitalization reports, traumatic injury and/or medical emergency associated with the mTBI were described as well. Traumatic injuries were categorized as facial and/or peripheral fractures (spine, limbs), moreover medical emergency associated with mTBI included infectious conditions (pneumopathy, urinary tract infection), rhabdomyolysis, renal failure, and/or a post-fall syndrome. In the absence of any traumatic or medical emergency and/or wound requiring suture, mTBI was classified as isolated-mTBI. Additionally, any alcohol intoxication was included in $\mathrm{g} / \mathrm{l}$.

\section{Outcome measure}

The primary outcome was an intracranial lesion found on a head CT-scan. As part of routine care, all head CTscans were interpreted by a senior neuro-radiologist who provided a written report. Intracranial lesions were described as follow: subarachnoid hemorrhages, acute subdural, intra-parenchymal hematoma and/or cerebral contusion. Cortical subcortical atrophy was also notified according to the neuro-radiologist's report.

\section{Sample size}

The estimated number of patients required with a $95 \%$ confidence interval with a $10 \%$ width was 365 patients, based on an hypothesis of $5 \%$ intracranial lesion prevalence $[4,9]$.

\section{Statistical analysis}

The intracranial lesion prevalence was described by frequency. Quantitative data were reported as an average 
with standard deviation (SD) or median with interquartile range (IQR) when the distribution was not normal. In univariate analysis, patients with and without intracranial lesion were compared with the Student's t-test for quantitative data and the Chi-squared test or the Fisher's exact test for qualitative data according to their respective conditions of use. Differences were considered significant if $p<0.05$. Relative risks with 95\% confidence interval were calculated for significant variables. Due to the sample size, a multivariate analysis was not performed. Statistical tests were conducted with Stata v11.2.

\section{Results}

Between January 2019 and June 2019, 365 patients were included. The average age of the entire cohort was $86.5( \pm$ 8.5 ) years old, ranging from 65 to 104, and 244 patients $(66.8 \%)$ were female. Overall, 141 patients $(38.6 \%)$ were receiving antiplatelet and $128(35.1 \%)$ anticoagulant medication. One hundred and twenty-six patients (34.5\%) presented cognitive impairment prior to ED visit.
The most common mTBI mechanism was groundlevel fall $(331 / 365,91.8 \%)$. Among the 7 patients $(1.9 \%)$ who had a road accident, 3 were drivers or passengers of a motor vehicle, 3 were cyclists and one was a pedestrian hit by a car. The mean blood alcohol level of the 7 inebriated patients was $2.2 \mathrm{~g} / \mathrm{l}(\mathrm{SD}=1.1)$.

Furthers characteristic of the study population with univariate analysis for intracranial lesions are presented in Table 1.

Median time between mTBI and ED arrival was 120 $\min (\mathrm{IQR}=90$ to 180$)$ and $142 \mathrm{~min}(\mathrm{IQR}=112$ to 180$)$ between ED arrival and the performance of head CTscan. Time of the mTBI event was unknown for $117 \mathrm{pa}-$ tients (32.1\%). The history of the injury and the clinical findings at ED are displayed in Table 2 with univariate analysis according to the presence of intracranial lesions.

Of the 365 patients included, 26 (7.1\%) had intracranial lesion. Compared with cutaneous frontal impact, the relative risk of intracranial lesion was 2.54 (95\% CI 1.20 to 5.42) for patients with temporoparietal or occipital

Table 1 Characteristics of patients presenting to the ED with mild traumatic brain injury and univariate analysis according to the presence of intracranial lesion

\begin{tabular}{|c|c|c|c|c|}
\hline & $\begin{array}{l}\text { Population } \\
(\boldsymbol{N}=365)\end{array}$ & $\begin{array}{l}\text { Intracranial lesion } \\
(\boldsymbol{N}=26)\end{array}$ & $\begin{array}{l}\text { No intracranial lesion } \\
(\boldsymbol{N}=339)\end{array}$ & $p$-value \\
\hline Age (years, SD) & $86.5(8.5)$ & $86.8(8.6)$ & $86.4(8.5)$ & 0.80 \\
\hline Age < $75(n, \%)$ & $41(11.2)$ & $2(7.7)$ & $39(11.5)$ & \\
\hline Age $\geq 75(n, \%)$ & $324(88.8)$ & $24(92.3)$ & $300(88.5)$ & 0.55 \\
\hline Gender, female (n, \%) & $244(66.8)$ & $22(84.6)$ & $222(65.5)$ & 0.05 \\
\hline \multicolumn{5}{|l|}{ Residence (n, \%) } \\
\hline Nursing home & $144(39.5)$ & $10(38.5)$ & $134(39.5)$ & 0.92 \\
\hline Home, with relatives & $115(31.5)$ & $9(34.6)$ & $106(31.3)$ & 0.72 \\
\hline Home, alone & $106(29.0)$ & $7(26.9)$ & $99(29.2)$ & 0.81 \\
\hline \multicolumn{5}{|l|}{ Antiplatelets (n, \%) } \\
\hline Aspirin & $112(30.7)$ & $11(42.3)$ & $101(29.8)$ & 0.18 \\
\hline Clopidogrel & $27(7.4)$ & $1(3.8)$ & $26(7.7)$ & 0.40 \\
\hline Aspirin + Clopidogrel & $2(0.55)$ & $0(0)$ & $2(0.59)$ & 0.86 \\
\hline \multicolumn{5}{|l|}{ Anticoagulant (n, \%) } \\
\hline Warfarin & $60(16.4)$ & $5(19.2)$ & $55(16.2)$ & 0.69 \\
\hline Direct oral anticoagulant & $61(16.7)$ & $3(11.5)$ & $58(17.1)$ & 0.34 \\
\hline Subcutaneous anticoagulant & $7(1.9)$ & $1(3.8)$ & $6(1.8)$ & 0.40 \\
\hline \multicolumn{5}{|l|}{ Psychoactive drug (n, \%) } \\
\hline Benzodiazepine & $100(27.4)$ & $10(38.4)$ & $90(26.5)$ & 0.19 \\
\hline Antidepressant & $65(17.8)$ & $8(30.8)$ & $57(16.8)$ & 0.07 \\
\hline Neuroleptic & $18(4.9)$ & $0(0.0)$ & $18(5.3)$ & 0.25 \\
\hline Antiepileptic & $11(3.0)$ & $0(0.0)$ & $11(3.2)$ & 0.43 \\
\hline \multicolumn{5}{|l|}{ History } \\
\hline Cognitive impairment $(n, \%)$ & $126(34.5)$ & $9(34.6)$ & $117(34.5)$ & 0.99 \\
\hline Clinical Frailty Scale (mean, SD) & $4.6(1.6)$ & $4.9(1.7)$ & $4.6(1.6)$ & 0.33 \\
\hline Neurosurgical intervention & $10(2.7)$ & $0(0.0)$ & $10(2.9)$ & 0.47 \\
\hline
\end{tabular}


Table 2 Injury history and clinical findings at the ED with univariate analysis according to the presence of intracranial lesion

\begin{tabular}{|c|c|c|c|c|}
\hline & $\begin{array}{l}\text { Population } \\
(\boldsymbol{N}=365)\end{array}$ & $\begin{array}{l}\text { Intracranial lesion } \\
(\boldsymbol{N}=26)\end{array}$ & $\begin{array}{l}\text { No intracranial lesion } \\
(\boldsymbol{N}=339)\end{array}$ & $p$-value \\
\hline Presence of witness & $101(27.7)$ & $7(26.9)$ & $94(27.7)$ & 0.92 \\
\hline \multicolumn{5}{|l|}{ Mechanisms of injury ( $n, \%)$} \\
\hline Ground-level fall & $335(91.8)$ & $25(96.1)$ & $310(92.3)$ & 0.40 \\
\hline Fall from $>1 \mathrm{~m}$ or 5 stairs & $14(3.8)$ & $1(3.9)$ & $13(3.6)$ & 0.65 \\
\hline Mechanical fall & $167(47.9)$ & $9(42.3)$ & $158(50.7)$ & 0.41 \\
\hline Unknow & $142(40.7)$ & $10(38.5)$ & $132(38.9)$ & 0.96 \\
\hline Faintness or vertigo & $27(7.7)$ & $3(11.5)$ & $24(7.1)$ & 0.30 \\
\hline Syncope & $13(3.7)$ & $2(7.7)$ & $11(2.3)$ & 0.26 \\
\hline Motor vehicle accident & $7(1.9)$ & $0(0.0)$ & $7(1.8)$ & 0.59 \\
\hline Unknown & $6(1.6)$ & $0(0.0)$ & $6(1.6)$ & 0.64 \\
\hline Head striking by an object & $3(0.9)$ & $0(0.0)$ & $3(0.7)$ & 0.80 \\
\hline \multicolumn{5}{|l|}{ Activity after falling (n, \%) } \\
\hline Getting up with assistance & $203(55.6)$ & $20(76.9)$ & $183(54.0)$ & 0.02 \\
\hline Staying on the floor for $>1 \mathrm{~h}$ & $108(29.6)$ & $4(15.4)$ & $104(30.7)$ & 0.1 \\
\hline Getting up alone & $54(14.8)$ & $2(7.7)$ & $52(15.3)$ & 0.29 \\
\hline \multicolumn{5}{|l|}{ Symptoms after injury ( $n, \%)$} \\
\hline Unknown & $49(13.5)$ & $2(7.7)$ & $47(14.0)$ & 0.37 \\
\hline Amnesia & $48(13.2)$ & $1(3.8)$ & $47(13.9)$ & 0.1 \\
\hline Headaches & $43(11.8)$ & $11(42.3)$ & $32(9.4)$ & $<0.001$ \\
\hline Loss of consciousness & $26(7.1)$ & $1(3.8)$ & $25(7.4)$ & 0.50 \\
\hline Vomiting & $11(3.0)$ & $3(11.5)$ & $8(2.4)$ & 0.004 \\
\hline Seizure & $2(0.6)$ & $0(0.0)$ & $2(0.6)$ & 0.9 \\
\hline
\end{tabular}

\section{Clinical examination}

Glasgow score:

15

14

13

Base skull fracture sign

Alcohol intoxication ( $\mathrm{n}, \%)$

Focal neurological sign

\section{Cutaneous injury (n, \%)}

Wound requiring suture

Hematoma

Cutaneous abrasion

No cutaneous lesion

Cutaneous impact location ( $n$, \%)

Frontal
Facial
Temporoparietal
No cutaneous impact
Occipital

\section{Associated traumatic and/or medical emergency (n, \%)}

$\begin{array}{ll}326(89.3) & 19(73.1) \\ 36(9.9) & 7(26.9) \\ 3(0.82) & 0(0) \\ 13(3.6) & 3(11.5) \\ 7(1.9) & 1(3.9) \\ 3(0.8) & 3(11.5)\end{array}$

$131(35.9)$

117 (32.0)

78 (21.4)

39 (10.7)

151 (41.4)

$86(23.4)$

$52(14.3)$

39 (10.7)

37 (10.1)
$13(50.0)$

$8(30.8)$

5 (20.0)

$0(0.0)$

10 (38.5)

1 (3.8)

8 (30.8)

0 (0)

7 (26.9)
307 (90.6)

0.005

$29(8.6)$

$3(0.9)$

$10(2.9)$

6 (1.8)

$0(0.0)$

118 (34.8)

109 (32.2)

73 (21.5)

40 (11.8)

$141(41.4)$

85 (25.0)

44 (13.0)

39 (11.8)

$30(8.8)$

130 (35.6)
0.002

0.80

0.06

0.41

$<0.001$

0.12

0.15

0.69

0.04

0.76

0.01

0.01

0.04

0.003 
Table 2 Injury history and clinical findings at the ED with univariate analysis according to the presence of intracranial lesion (Continued)

\begin{tabular}{|c|c|c|c|c|}
\hline & $\begin{array}{l}\text { Population } \\
(\boldsymbol{N}=365)\end{array}$ & $\begin{array}{l}\text { Intracranial lesion } \\
(\boldsymbol{N}=26)\end{array}$ & $\begin{array}{l}\text { No intracranial lesion } \\
(\boldsymbol{N}=339)\end{array}$ & $p$-value \\
\hline Traumatic injury & $71(19.5)$ & $5(19.2)$ & $66(19.5)$ & 0.97 \\
\hline Spine or limb fracture & $39(10.7)$ & $4(15.4)$ & $35(10.3)$ & 0.30 \\
\hline Facial bones fracture & $9(2.5)$ & $1(3.9)$ & $8(2.4)$ & 0.19 \\
\hline Medical emergency & $58(8.8)$ & $2(7.7)$ & $56(16.5)$ & 0.24 \\
\hline
\end{tabular}

impact and 0.12 (95\% CI 0.01 to 0.93$)$ for patients with cutaneous facial impact or no cutaneous impact. Relative risk of intracranial lesions according to the cutaneous impact location are presented on Fig. 1. There was no statistical increase in risk of intracranial lesion in patients receiving antiplatelets $(\mathrm{RR}=1.43 ; 95 \% \mathrm{CI} 0.68$ to 2.99 ) or anticoagulants ( $R R=0.98 ; 95 \%$ CI 0.45 to 2.14 ). The univariate analysis of relative risk for intracranial lesions are displayed on Table 3.

Among the 26 patients with intracranial lesions, 3 (11.5\%) had a focal deficit: one presented aphasia (3.8\%), one homonymous hemianopsia (3.8\%), and one hemiparesis $(3.8 \%)$. Thirteen patients $(50 \%)$ showed a subdural hematoma, 9 (34.6\%) had subarachnoid hemorrhage, and $4(15.4 \%)$ an intraparenchymal hematoma. No patient required neurosurgical intervention. Eighteen patients $(69.2 \%)$ with intracranial lesion were hospitalized and $2(7.7 \%)$ were transferred to the intensive care unit. Warfarin effects were reversed by prothrombin complex concentrate and vitamin $\mathrm{K}$ in 5 patients (19.2\%). One patient treated with antivitamin $\mathrm{K}$ had an extensive intraparenchymal hematoma despite reversion and died 2 days after admission.

Eighty-nine patients presented cortical subcortical atrophy on head CT-scans; 8 of them $(9.0 \%)$ had an intracranial lesion $(p=0.43)$.
Overall, 106 patients (29.0\%) were hospitalized and 141 (38.6\%) had an isolated mTBI.

Patients' characteristics, injury history, clinical findings and outcomes according to cutaneous impact location according to the cutaneous impact location are presented in the Table 4.

\section{Discussion}

Our mTBI-related intracranial lesion prevalence of $7.2 \%$ was consistent with recent studies performed in the elderly $[9,13]$. This lower prevalence found in EDs recent studies may have several causes. Firstly, current guidelines recommend a head CT-scan in most patients over 65 years old after mTBI. Thus, this large indication may decrease intracranial lesion prevalence among this population. Secondly, according to the mTBI definition of 1993, injury mechanisms were not only direct head strikes but also acceleration/deceleration movements without any direct external trauma of the head [14]. In accordance with literature our findings showed that ground-level fall was the most common cause of mTBI $[1,13]$. Therefore, the amount of mTBI kinetics involved in ground level fall in the elderly is less than in younger populations (ie: falls from over one meter or motor vehicle accidents). Thus, ground-level fall, especially without head impact, may not

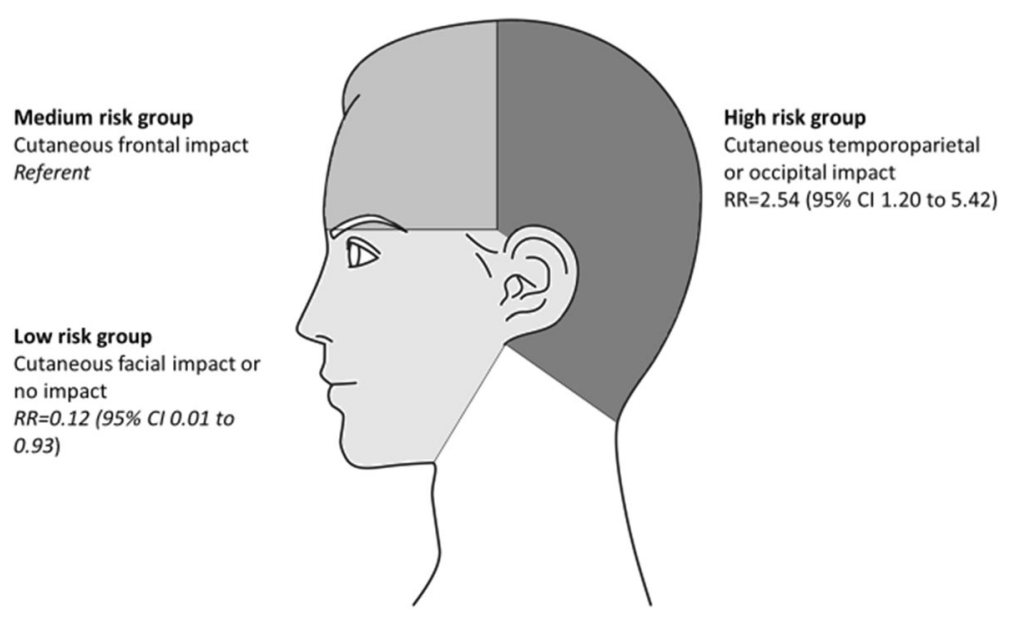

Fig. 1 Univariate relative risk of intracranial injury according to the cutaneous impact location 
Table 3 Univariate analysis of relative risk for intracranial lesion after mTBI among patients aged of 65 years old and over presenting to the ED

\begin{tabular}{|c|c|c|c|}
\hline & Relative risk & $95 \% \mathrm{Cl}$ & $p$-value \\
\hline \multicolumn{4}{|l|}{ Sex } \\
\hline Male & 1.00 & & \\
\hline Female & 2.76 & $0.98-7.84$ & 0.043 \\
\hline \multicolumn{4}{|l|}{ Getting up with assistance } \\
\hline Getting up alone & 1.00 & & \\
\hline Getting up with assistance & 2.66 & $1.09-6.47$ & 0.023 \\
\hline \multicolumn{4}{|l|}{ Cutaneous impact location } \\
\hline Frontal & 1.00 & & \\
\hline Temporoparietal or Occipital & 2.54 & $1.19-5.42$ & 0.012 \\
\hline Facial or no cutaneous impact & 0.12 & $0.01-0.93$ & 0.014 \\
\hline \multicolumn{4}{|l|}{ Headaches } \\
\hline No headaches & 1.00 & & \\
\hline Headaches & 5.49 & $2.70-11.17$ & $<0.001$ \\
\hline \multicolumn{4}{|l|}{ Vomiting } \\
\hline No vomiting & 1.00 & & \\
\hline Vomiting & 4.19 & $1.48-11.91$ & 0.008 \\
\hline \multicolumn{4}{|l|}{ Focal neurological sign } \\
\hline No focal neurological sign & 1.00 & & \\
\hline Focal neurological sign & 15.74 & $10.59-23.38$ & $<0.001$ \\
\hline \multicolumn{4}{|l|}{ Glasgow } \\
\hline 15 & 1.00 & & \\
\hline 14 & 3.34 & $1.51-7.39$ & 0.002 \\
\hline
\end{tabular}

have enough kinetics to induce intracranial lesion in the elderly.

Compared with cutaneous frontal impact, temporoparietal and occipital impact had an intracranial lesion relative risk of 2.54 (95\% CI 1.19 to 5.42 ). To our knowledge, the cutaneous impact location in the context of mTBI in elderly has not been assessed. Several hypotheses could explain these results. The musculature reduction of the trunk and neck, to which ageing of the individual contributes, could increase the force of temporoparietal or occipital impact in the event of ground-level fall [4, 15]. Conversely, in case of frontal impact, some of this kinetic energy may be reduced by postural adaptation reflexes of the upper limbs. In addition, frontal impact may have less risk of intracranial lesion due to the fact the frontal bone is thicker than temporal, parietal and occipital bones, [16]. Moreover, in our study and according to literature, 50\% of intracranial injuries were subdural hemorrhages $[4,13]$. The pathophysiology of this hemorrhage as Miller JD et al. reminds us, involves a direct trauma with low kinetic energy able to affect the venous network of the dura mater and the arachnoid [17]. Furthermore, several hypotheses involving the vulnerability of vascular tissue and age- related white matter alterations have been put forward to explain the susceptibility of old patients to hemorrhage subsequent to a direct trauma with low kinetic energy [18, 19]. The cutaneous impact location in a context of mild traumatic brain injury has already been studied among children. In a recent large study of 3866 children younger than 17 years old, Burns EC et al., [20] showed that temporal/parietal and occipital impact location had significantly greater odds of intracranial lesions than other impact locations. Furthermore, these odds were greatest in children aged from 0 to 6 months. The mechanisms involved in youngest children and frailty elderly might be the same: despite the low kinetic energy, the absence of postural adaptation reflexes of the upper limbs may trigger intracranial lesions especially in case of temporal/parietal and occipital impact. Taken together, these findings could explain why patients with cutaneous temporoparietal or occipital impact had a higher risk of intracranial lesion than those with cutaneous frontal impact. Thus, to better apprehend the relevance of head CT-scan in the context of ground-level fall with mTBI among the elderly, risk groups of intracranial lesion may be identified regarding the cutaneous impact location as follow: low risk groups (with facial or no cutaneous impact), medium risk (cutaneous frontal impact) and high risk (cutaneous temporoparietal and occipital impact). This objective sign may help us to better assess the risk of intracranial lesions and better target head CT-scan indication.

In spite of the prospective nature of our study, it was not possible to determine the fall cause in more than one third of the cases. Timler et al. showed in their retrospective study that the mTBI mechanism was not identified in $23.6 \%$ of the cases [13]. Furthermore, in our study, symptoms after mTBI were unknown in $13.5 \%$ of the cases. Not only the symptom sensitivity for intracranial lesion diagnosis are low, but the occurrence of those are also often unknown by the physician in charge of the patient $[7,13,21]$. These results strengthen the hypothesis of Papa et al. suggesting that the term mTBI is sometimes misused in the elderly [7]. However, clinical findings as headaches $(\mathrm{RR}=5.49$; 95\% CI 2.70-11.17), vomiting and focal neurological signs $(R R=15.74 ; 95 \% C I$ 10.59-23.37) were significantly associated with the development of intracranial lesions. Regarding the Glasgow score, a score of 14 was significantly associated with intracranial lesion $(R R=3.34$; 95\% CI 1.51-7.39). Literature shows divergent findings; on the one hand, some studies discredited Glasgow score in elderly by showing less sensitive than in younger patients to detect intracranial lesion [22]. On the other hand, some studies suggested that a Glasgow score limit of 14 instead of 13 may improve sensitivity to predict poor outcome in elderly [23].

Ongoing treatments with antiplatelets or anticoagulants were not associated with a significant increase in 
Table 4 Patients' characteristics, injury history, clinical findings and outcomes according to the cutaneous impact location with univariate analysis

\begin{tabular}{|c|c|c|c|c|}
\hline & $\begin{array}{l}\text { Low risk group } \\
(\boldsymbol{N}=125)\end{array}$ & $\begin{array}{l}\text { Medium risk group } \\
(\boldsymbol{N}=151)\end{array}$ & $\begin{array}{l}\text { High risk group } \\
(\boldsymbol{N}=89)\end{array}$ & $\boldsymbol{P}$ value \\
\hline Age (years, SD) & $87.8(7.9)$ & $85.7(8.3)$ & $85.9(9.3)$ & 0.28 \\
\hline Clinical Frailty scale (mean, SD) & $4.6(1.6)$ & $4.7(1.7)$ & $4.4(1.6)$ & 0.98 \\
\hline Residence (n, \%) & & & & 0.22 \\
\hline Nursing home & $57(45.6)$ & $55(36.4)$ & $32(35.9)$ & \\
\hline Home & $68(54.4)$ & $96(63.6)$ & $57(64.1)$ & \\
\hline \multicolumn{5}{|l|}{ Medication (n, \%) } \\
\hline Antiplatelet & $46(28.8)$ & $58(38.4)$ & $33(37.1)$ & 0.96 \\
\hline Anticoagulant & 47 (37.6) & $50(33.1)$ & $31(34.8)$ & 0.74 \\
\hline Psychoactive drugs & $52(41.6)$ & $57(37.8)$ & $29(32.6)$ & 0.41 \\
\hline Ground-level fall (n, \%) & & & & 0.31 \\
\hline Mechanical fall & $53(42.4)$ & $73(48.3)$ & $38(42.7)$ & \\
\hline Unknow & $49(39.2)$ & $54(35.8)$ & $30(33.7)$ & \\
\hline Faintness or vertigo & $9(7.2)$ & $6(4.0)$ & $11(12.4)$ & \\
\hline Syncope & $3(2.4)$ & $6(4.0)$ & $3(3.4)$ & \\
\hline \multicolumn{5}{|l|}{ Symptoms after injury (n, \%) } \\
\hline Unknown & $18(14.4)$ & $22(14.6)$ & $6(6.7)$ & 0.16 \\
\hline Amnesia & $20(16.0)$ & $15(9.9)$ & $13(14.6)$ & 0.29 \\
\hline Headache & $13(10.4)$ & $12(7.9)$ & $10(11.2)$ & 0.01 \\
\hline Loss of consciousness & $12(9.6)$ & $6(3.9)$ & $8(9.0)$ & 0.14 \\
\hline Vomiting & $4(3.2)$ & $1(0.7)$ & $6(6.7)$ & 0.03 \\
\hline Seizure & 0 & $1(0.7)$ & 0 & 0.53 \\
\hline \multicolumn{5}{|l|}{ Clinical examination ( $n, \%)$} \\
\hline Glasgow score & & & & 0.1 \\
\hline 15 & $112(89.6)$ & $132(87.4)$ & $82(92.1)$ & \\
\hline 14 & $10(8.0)$ & 19 (12.6) & $7(7.9)$ & \\
\hline 13 & $3(2.4)$ & 0 & 0 & \\
\hline Wound requiring suture & $28(22.4)$ & $68(45.0)$ & $35(39.3)$ & $<0.001$ \\
\hline Hematoma & $38(30.4)$ & $49(32.5$ & $32(35.9)$ & 0.7 \\
\hline Cutaneous abrasion & $21(18.8)$ & $34(25.0)$ & $22(26.2)$ & 0.38 \\
\hline Intracranial lesion (n, \%) & & & & 0.16 \\
\hline Subdural hematoma & $1(0.8)$ & $2(1.3)$ & $10(11.2)$ & \\
\hline Subarachnoid hemorrhage & 0 & $6(4.0)$ & $3(3.4)$ & \\
\hline Intraparenchymal hematoma & 0 & $2(1.3)$ & $2(2.2)$ & \\
\hline Outcome (n, \%) & & & & 0.51 \\
\hline Hospitalization & $33(26.4)$ & $42(27.8)$ & $29(32.6)$ & \\
\hline ICU & $1(0.8)$ & $1(0.7)$ & 0 & \\
\hline
\end{tabular}

the risk of intracranial lesion. Recent studies suggest that the use of antiplatelet alone does not increase the risk of mTBI-related intracranial lesion [24]. About anticoagulant, especially in ground-level fall context, recent studies suggest an increasing risk of intracranial lesion but not a worsen morbidity or mortality $[25,26]$. These results should be analyzed carefully, despite the absence of multivariate analysis performed in our study due to the small sample size of it. Moreover, even if ICL prevalence was similar we still notice a higher prevalence in the Aspirin group with $9.8 \%(11 / 112)$ and $8.3 \%$ in the Warfarin group with $8.3 \%(5 / 60)$ than the overall population (7.2\%, 26/365). 
Contrary to our initial hypothesis, patients' frailty assessed by the Clinical Frailty Scale was not associated with a significant increase in the risk of developing intracranial lesion. However, the frailty measured by this scale was high in our population (mean 4.6/7; SD 1.6). This finding is consistent with literature suggesting that frailty is associated with an high incidence of mTBI [15]. Nonetheless, getting up with assistance was associated with great risk of intracranial lesion $(\mathrm{RR}=2.66$; $95 \% \mathrm{IC}$ 1.09-6.47). This may reflect declined functional abilities in these patients who might have impaired postural adaptive reflexes.

Additionally, we identified the same risk factors for intracranial lesion classically identified in older patients such as female ( $\mathrm{RR}=2.76$; 95\% CI $0.98-7.83)$ [21]. The age population average was high. Due to this Fournier et al. suggest increasing the age limit from 65 to 75 years-old. The fact of adjusting the age limit to 75 yearold in the Canadian head CT rule may reduce head CTscans performed of $25 \%$ without any intracranial lesion missed [27]. In their retrospective cohort, Riccardi et al. even suggested to increase this limit to 80 years old [9].

Hospitalization rate in our cohort was low (29\%). This is consistent with literature and reinforces recent hypotheses of potentially avoidable and/or inappropriate use of emergency services, particularly for the $\mathrm{NH}$ residents counting for $39.5 \%$ of our cohort [13]. The following term: 'potentially avoidable and/or inappropriate use of emergency services' has no consensual definition, but may represent almost half of the transfers to the ED from nursing homes [28]. Better target intracranial lesions risk after mTBI among this population may reduce the need for ED visits. Our study further strengthens this theory since $38.7 \%$ of the patients admitted to the ED had an isolated mTBI, without wounds requiring suture or any medical and/or trauma emergency care.

The strength of our prospective study relies on the anamnesis accuracy and clinical findings in the ED since it was a real-life observational study. This prospective inclusion is a true reflection of the semiological survey conducted by ED practitioners. The main limitation of our study was the population's size. Its main objective was to establish the prevalence of intracranial lesion and it may lack power to identify certain risk factors. Furthermore, the number of cases was not sufficient to conduct a multivariate analysis. In addition, there was no patient follow-up while according to literature, there might be an increased risk of delayed hemorrhage especially in patients treated with anticoagulants [29]. Moreover, the readmission rate to hospital within 1 month after an ED visit for mTBI-related intracranial lesions might be high, particularly for the elderly with fallrelated mTBI [30, 31].

\section{Conclusions}

To sum up, the prevalence of mTBI-related intracranial lesion in elderly patients admitted to the ED was similar to younger patients. This finding might be partly explained by the low kinetic energy and the pathophysiology of hemorrhage in the context of ground-level fall. These results suggest that the cutaneous impact location may help to identify risk for mTBI-related intracranial lesions in older patients. A prospective, multicenter ED study would be useful to confirm these potential risk factors.

\section{Abbreviations}

CT-scan: Computed Tomography scan; ED: Emergency Department; NH: Nursing Home; mTBI: Mild Traumatic Brain Injury

\section{Acknowledgments}

We would like to thank all emergency practitioners for their involvement in this prospective study and Ana Inés Darquier for helping us translate this manuscript into English.

\section{Authors' contributions}

$X D, F B, E S, M H, S C, C H$ and $D L$ set up the study design. Data acquisition was performed by XD. XD, FB, CH and DL analyzed and interpreted the patients' data. XD drafted the manuscript. All authors read and approved the final manuscript.

\section{Funding}

This study wasn't supported by any funding.

\section{Availability of data and materials \\ The datasets generated during the current study are available from the corresponding author on reasonable request.}

\section{Ethics approval and consent to participate}

Patients were informed that their data will be used for the study. The University Hospital signed a commitment of compliance to the reference methodology MR-004 of the National Commission for Informatics and Liberties (CNIL number: RnIPH 2019-82). This study was approved by the University Hospital and confirm that all ethic requirements were totally respected herein.

\section{Competing interests}

The authors declare that they have no competing interests.

\section{Author details}

${ }^{1}$ Emergency Department, Centre Hospitalo-Universitaire de Toulouse, Toulouse, France. ${ }^{2}$ Department of Neurosurgery, Centre

Hospitalo-Universitaire de Toulouse, Toulouse, France. ${ }^{3}$ Department of Geriatric Medecine, Centre Hospitalo-Universitaire de Toulouse, Toulouse, France.

Received: 13 May 2020 Accepted: 27 August 2020

Published online: 31 August 2020

\section{References}

1. Hawley C, Sakr M, Scapinello S, Salvo J, Wrenn P. Traumatic brain injuries in older adults-6 years of data for one UK trauma Centre: retrospective analysis of prospectively collected data. Emerg Med J. 2017;34(8):509-16.

2. Faul $M, X u L$, Wald MM, Coronado V, Dellinger AM. Traumatic brain injury in the United States: national estimates of prevalence and incidence, 20022006. Inj Prev. 2010;16(Suppl 1):A268.

3. Thompson HJ, McCormick WC, Kagan SH. Traumatic brain injury in older adults: epidemiology, outcomes, and future implications. J Am Geriatr Soc 2006;54(10):1590-5.

4. Gardner RC, Dams-O'Connor K, Morrissey MR, Manley GT. Geriatric traumatic brain injury: epidemiology, outcomes, knowledge gaps, and futuredirections. J Neurotrauma. 2018;35(7):889-906. 
5. Undén J, Ingebrigtsen T, Romner B. Scandinavian guidelines for initial management of minimal, mild and moderate head injuries in adults: an evidence and consensus-based update. BMC Med. 2013;11:50.

6. Stiell IG, Wells GA, Vandemheen K, Clement C, Lesiuk H, Laupacis A, et al. The Canadian CT head rule for patients with minor head injury. Lancet. 2001;357(9266):1391-6.

7. Papa L, Mendes ME, Braga CF. Mild traumatic brain injury among the geriatric population. Curr Transl Geriatr Exp Gerontol Rep. 2012;1(3):135-42.

8. Caterino JM, Brown NV, Hamilton MW, Ichwan B, Khaliqdina S, Evans DC, et al. Effect of geriatric-specific trauma triage criteria on outcomes in injured older adults, a statewide retrospective cohort study. J Am Geriatr Soc. 2016; 64(10):1944-51.

9. Riccardi A, Frumento F, Guiddo G, Spinola MB, Corti L, Minuto P, et al. Minor head injury in the elderly at very low risk: a retrospective study of 6 years in an emergency department (ED). Am J Emerg Med. 2013;31(1):37-41.

10. Yuan M-K, Tsai D-C, Chang S-C, Yuan M-C, Chang S-J, Chen H-W, et al. The risk of cataract associated with repeated head and neck CT studies: a nationwide population-based study. AJR Am J Roentgenol. 2013;201(3):62630 .

11. SFMU, Jehlé E, Honnart D, Grasleguen C, Bouget J, Dejoux C, et al. Traumatisme crânien léger (score de Glasgow de 13 à 15) : triage, évaluation, examens complémentaires et prise en charge précoce chez le nouveau-né, l'enfant et l'adulte: Société française de médecine d'urgence. Ann Françaises Méd D’urgence. 2012;2(3):199-214.

12. Juma $\mathrm{S}$, Taabazuing M-M, Montero-Odasso M. Clinical frailty scale in an acute medicine unit: a simple tool that predicts length of stay. Can Geriatr J. 2016;19(2):34-9.

13. Timler D, Dworzyński MJ, Szarpak Ł, Gaszyńska E, Dudek K, Gałązkowski R. Head trauma in elderly patients: mechanisms of injuries and CT findings. Adv Clin Exp Med. 2015;24(6):1045-50.

14. Head Injury Interdisciplinary Special Interest Group of the American Congress of Rehabilitation Medicine. Definition of mild traumatic brain injury. J Head Trauma Rehabil. 1993;8(3):86.

15. Dams-O'Connor K, Gibbons LE, Landau A, Larson EB, Crane PK. Health problems precede traumatic brain injury in older adults. J Am Geriatr Soc. 2016;64(4):844-8.

16. Murty O. Variability in thickness of skull bones and sternum. J Forensic Med Toxicol. 2009;26(2):26-31

17. Miller JD, Nader R. Acute subdural hematoma from bridging vein rupture: a potential mechanism for growth: clinical article. J Neurosurg. 2014;120(6): $1378-84$.

18. Ikonomovic MD, Mi Z, Abrahamson EE. Disordered APP metabolism and neurovasculature in trauma and aging: combined risks for chronic neurodegenerative disorders. Ageing Res Rev. 2017;34:51-63.

19. Liu H, Yang Y, Xia Y, Zhu W, Leak RK, Wei Z, et al. Aging of cerebral white matter. Ageing Res Rev. 2017;34:64-76.

20. Burns ECM, Grool AM, Klassen TP, Correll R, Jarvis A, Joubert G, et al. Scalp hematoma characteristics associated with intracranial injury in pediatric minor head injury. Acad Emerg Med. 2016;23(5):576-83.

21. Styrke J, Stålnacke B-M, Sojka P, Björnstig U. Traumatic brain injuries in a well-defined population: epidemiological aspects and severity. J Neurotrauma. 2007;24(9):1425-36

22. Salottolo K, Levy AS, Slone DS, Mains CW, Bar-Or D. The effect of age on Glasgow coma scale score in patients with traumatic brain injury. JAMA Surg. 2014;149(7):727-34

23. Caterino JM, Raubenolt A, Cudnik MT. Modification of Glasgow coma scale criteria for injured elders. Acad Emerg Med. 2011;18(10):1014-21.

24. Ganetsky M, Lopez G, Coreanu T, Novack V, Horng S, Shapiro NI, et al. Risk of intracranial hemorrhage in ground-level fall with antiplatelet or anticoagulant agents. Acad Emerg Med. 2017;24(10):1258-66.

25. Probst MA, Gupta M, Hendey GW, Rodriguez RM, Winkel G, Loo GT, et al. Prevalence of intracranial injury in adult patients with blunt head trauma with and without anticoagulant or antiplatelet use. Ann Emerg Med. 2020 Mar 1;75(3):354-64

26. Batey M, Hecht J, Callahan C, Wahl W. Direct oral anticoagulants do not worsen traumatic brain injury after low-level falls in the elderly. Surgery. 2018;164(4):814-9.

27. Fournier N, Gariepy C, Prévost J-F, Belhumeur V, Fortier É, Carmichael P-H, et al. Adapting the Canadian $C T$ head rule age criteria for mild traumatic brain injury. Emerg Med J. 2019;36(10):617-9.
28. Perrin A, Tavassoli N, Mathieu C, Hermabessière S, Houles M, McCambridge $C_{\text {, et }}$ al. Factors predisposing nursing home resident to inappropriate transfer to emergency department. The FINE study protocol. Contemp Clin Trials Commun. 2017:7:217-23.

29. Marcia L, Moazzez A, Plurad DS, Putnam B, Kim DY. Utility of repeat head CT in patients on preinjury antithrombotic medications. Am Surg. 2018;84(10): 1626-9.

30. Brito A, Costantini TW, Berndtson AE, Smith A, Doucet JJ, Godat LN. Readmissions after acute hospitalization for traumatic brain injury. J Surg Res. 2019;244:332-7.

31. Hoffman GJ, Liu H, Alexander NB, Tinetti M, Braun TM, Min LC. Posthospital fall injuries and 30-day readmissions in adults 65 years and older. JAMA Netw Open. 2019;2(5):e194276.

\section{Publisher's Note}

Springer Nature remains neutral with regard to jurisdictional claims in published maps and institutional affiliations.
Ready to submit your research? Choose BMC and benefit from:

- fast, convenient online submission

- thorough peer review by experienced researchers in your field

- rapid publication on acceptance

- support for research data, including large and complex data types

- gold Open Access which fosters wider collaboration and increased citations

- maximum visibility for your research: over $100 \mathrm{M}$ website views per year

At BMC, research is always in progress.

Learn more biomedcentral.com/submissions 